\title{
OPTIMAL DIVIDEND POLICY WHEN CASH RESERVES FOLLOW A JUMP-DIFFUSION PROCESS UNDER MARKOV-REGIME SWITCHING
}

\author{
ZHENGJUN JIANG, ${ }^{*}$ Beijing Normal University - \\ Hong Kong Baptist University United International College
}

\begin{abstract}
In this paper we study the optimal dividend payments for a company of limited liability whose cash reserves in the absence of dividends follow a Markov-modulated jumpdiffusion process with positive drifts and negative exponential jumps, where parameters and discount rates are modulated by a finite-state irreducible Markov chain. The main aim is to maximize the expected cumulative discounted dividend payments until bankruptcy time when cash reserves are nonpositive for the first time. We extend the results of Jiang and Pistorius [15] to our setup by proving that it is optimal to adopt a modulated barrier strategy at certain positive regime-dependent levels and that the value function can be explicitly characterized as the fixed point of a contraction.
\end{abstract}

Keywords: Optimal dividend policy; Markov chain; regime switching; jump-diffusion process; stochastic control

2010 Mathematics Subject Classification: Primary 93E20

Secondary 91B70; 60H30

\section{Introduction}

A classical research topic in financial and actuarial mathematics is optimal dividend distribution for a company, which can be phrased as the tradeoff between the optimal timing and the sizes of dividend payments in the presence of bankruptcy risk, when the objective of the company is to maximize the expected value of the cumulative discounted dividend payments until bankruptcy. The earliest work in this setting can be traced back to De Finetti [9] under the binomial model. In continuous time the problem was posed and solved in a Brownian motion model for the cash reserves by Jeanblanc-Piqué and Shiryaev [14], and Asmussen and Taksar [1], using optimal control theory. Since then an extensive amount of literature has appeared on the dividend problem and its various extensions (see, e.g. [2], [5], [6], [8], [16], [17], and [18]).

Generally speaking, the specific form of the optimal dividend policy has been found to depend on the expected growth rate and the variability of future revenues, and the discount rate. These quantities are of great interest to us and will evolve in a time reflecting changing market and economic conditions, and those changes may happen gradually or occur abruptly and be more substantial. In this paper we will focus on the changes of the latter type (also called regime shifts or switches), and model cash reserves or risk reserves in the absence of dividends as a Markov-modulated jump-diffusion process. The underlying motivations are that premium per unit of time and discount rate may vary with the state of the economy and interest rate, that

Received 19 April 2013; revision received 25 February 2014.

* Postal address: Beijing Normal University - Hong Kong Baptist University United International College, 28 Jinfeng Road, Tangjiawan, Zhuhai, 519085, P. R. China. Email address: zhengjunjiang@uic.edu.hk. 
loss size may follow different probability distributions in different states of the economy, and that the optimal dividend policy as a Markov-modulated jump-diffusion process has not been discussed in detail in the literature.

Since Hamilton [11] and [12], a substantial econometric literature has appeared that supports the use of Markov regime-switching models to describe business cycles, term structure of interest rates, and other macroeconomic quantities. Such models have been shown to be capable of capturing occasional simultaneous and substantial changes of the parameters. Regimeswitching models also have the advantage of retaining a degree of analytical tractability, and models from this class can in principle approximate a given diffusion arbitrarily closely by taking the state space large enough and specifying the generator matrix appropriately. Such an application was developed by Zhu and Chen [25]. Different dividend optimization problems, but still in a regime-switching setting, have been recently treated by Sotomayor and Cadenillas [20], Wei et al. [21], [22], [23], and Zhu [24].

To the best of the author's knowledge, Jiang and Pistorius [15] is the earliest paper in which a fixed point theorem is used to prove the existence of optimal dividend barriers and the corresponding value function under Markov-regime switching in the cases of not only positive but also negative drifts. However, the existing literature does not cover optimal dividend payments in a setting of a Markov-modulated jump-diffusion process with parameters modulated by a finite state Markov chain and discount rate modelled as a deterministic function of the current state of the chain, which is our research focus and a natural extension of Belhaj [5] and Jiang and Pistorius [15].

In this setting, we will consider the problem of the management of the company to find a dividend policy that maximizes the expected discounted dividend payments until bankruptcy, which is defined to occur at the first moment when the level of the cash reserves hits or is below 0 . We will restrict ourselves to the case where the management can only control the timing and the size of the dividend payments. Our main aim is to prove by following the similar arguments of Jiang and Pistorius [15] that, in the case of positive drifts in all regimes, it is also optimal to adopt a barrier strategy at certain positive regime-dependent levels and to provide an explicit characterization of the value function as the fixed point of a contraction. The remainder of the paper is organized as follows. In Section 2 we provide a problem formulation, introduce scale functions, and present lower and upper bounds of value function. In Section 3 we present a dynamic programming equation, the corresponding optimal divided strategy, and related theorems. In Section 4 we construct and prove an iterative algorithm in order to calculate the value function $V$ and the corresponding optimal dividend barriers in different regimes. In Section 5 we conclude with a brief summary of this paper.

\section{Problem formulation}

Let $\left\{W_{t}: t \geq 0\right\}$ be a Wiener process, $\left\{N_{t}: t \geq 0\right\}$ be an independent Poisson process with intensity $\lambda>0$, and $\left\{Z_{t}: t \geq 0\right\}$ be a continuous time irreducible Markov chain with finite state space $E$ and generator matrix $Q=\left(q_{i j}\right)_{i, j \in E}$ with $\sum_{j \in E} q_{i j}=0$, independent of $W$ and $N$. Assume that cash reserves $X=\left\{X_{t}, t \geq 0\right\}$ of a financial corporation with limited liability follow, in the absence of dividend payments, a Markov-modulated jump-diffusion process with positive drifts and negative exponential jumps, that is, $X$ satisfies the following stochastic differential equation (SDE)

$$
\mathrm{d} X_{t}=\mu\left(Z_{t}\right) \mathrm{d} t+\sigma\left(Z_{t}\right) \mathrm{d} W_{t}-\mathrm{d} J_{t}, \quad X_{0}=x>0, Z_{0}=i,
$$


where $J_{t}$ is a suitabe regime-switching compound Poisson process and (2.1) is equivalent to the following stochastic integral representation

$$
X_{t}=x+\int_{0}^{t} \mu\left(Z_{s}\right) \mathrm{d} s+\int_{0}^{t} \sigma\left(Z_{s}\right) \mathrm{d} W_{s}-\sum_{k=1}^{N_{t}} Y_{k}\left(Z_{t_{k}}\right), \quad Z_{0}=i,
$$

where $Z$ represents the state of the economy and $t_{k}$ is the time of the $k$ th stochastic positive jump $Y_{k}(j)$ for $Z_{t_{k}}=j \in E$ such that $0<t_{1}<t_{2}<\cdots<t_{N_{t}} \leq t$, the jump size $Y_{k}(j)$, independent of $N$ and $W$, has exponential distribution with mean $1 / \delta_{j}>0$ for every state $j \in E$. For every state $i$ in $E$, both drift parameter $\mu(i)>0$ and volatility parameter $\sigma(i)>0$ are assumed to be known constants. In case there is no notational confusion possible, we will write $\mu_{i}$ and $\sigma_{i}$ for $\mu(i)$ and $\sigma(i)$, respectively. The processes $X$ and $Z$ are defined on some filtered probability space $(\Omega, \mathcal{F}, \boldsymbol{F}, \mathbb{P})$, where $\boldsymbol{F}=\left\{\mathcal{F}_{t}, t \geq 0\right\}$ denotes the right-continuous completed filtration jointly generated by $X$ and $Z$. We denote by $\mathbb{P}_{x, i}$ and $\mathbb{P}_{x}$ the measure $\mathbb{P}$ conditioned on $\left\{X_{0}=x, Z_{0}=i\right\}$ and $\left\{X_{0}=x\right\}$, respectively, and write $\mathbb{E}_{x, i}$ and $\mathbb{E}_{x}$ for the corresponding expectations. We assume that the processes $X$ and $Z$ are both fully observable to the shareholders, and that they decide on the dividend strategies on the basis of the available information.

A dividend strategy $D$ is a nondecreasing and left-continuous stochastic process $D=$ $\left\{D_{t}: t \geq 0\right\}$ with $D_{0-}=0$, where $D_{t}$ represents the cumulative dividends paid out until time $t$. It is also assumed that the dividend payments reduce the reserves but have no effect on the business and that there are no transaction costs associated with the payment of dividends. The dynamics of the risk reserve process $U=\left\{U_{t}: t \geq 0\right\}$ in the presence of dividend payments are then represented by

$$
\mathrm{d} U_{t}=\mathrm{d} X_{t}-\mathrm{d} D_{t}
$$

for all $t$ until the time $\tau$ of bankruptcy and $\mathrm{d} U_{t}=0$ for $t$ after $\tau$, where

$$
\tau=\inf \left\{t \geq 0: U_{t} \leq 0\right\}
$$

is the first time that $U$ hits or is below 0 . To avoid degeneracies only those dividend strategies will be considered that have no lump sum dividend payments larger than the current level of the reserves: A dividend strategy $D$ is called admissible if $D$ is $\boldsymbol{F}$-adapted, $\mathrm{d} D_{t}=0$ for $t \geq \tau$ and

$$
U_{t} \geq D_{t}-D_{t-} \text { for all } t<\tau \text {. }
$$

Denote by $\mathscr{D}$ the set of admissible dividend strategies and define the objective function of the shareholders as the following value function $V(x, i)$ in regime $i$,

$$
V(x, i)=\sup _{D \in \mathcal{D}} V_{D}(x, i),
$$

where $V_{D}$ denotes the expected value of the discounted dividends until the time of ruin $\tau$ under the dividend strategy $D$,

$$
V_{D}(x, i)=\mathbb{E}_{x, i}\left[\int_{0}^{\tau} \exp \left(-\int_{0}^{t} r\left(Z_{s}\right) \mathrm{d} s\right) \mathrm{d} D_{t}\right],
$$

with $r: E \rightarrow(0, \infty)$ the Markov-modulated rate of discounting. The problem for the shareholders is to identify a dividend strategy $D^{*} \in \mathscr{D}$ that attains the supremum in (2.4), that is, $V \equiv V_{D^{*}}$. 


\subsection{Scale functions}

Scale functions play a crucial role in expressing the value function $V(x, i)$ in $(2.4)$ for the regime $i$. In the absence of regime switching, $X_{t}$ following (2.2) with initial regime $i$ has its Laplace exponent $\psi_{i}(\theta)$ given by

$$
\psi_{i}(\theta):=\frac{1}{t} \log E\left[\mathrm{e}^{\theta\left(X_{t}-X_{0}\right)}\right]=\frac{1}{2} \sigma_{i}^{2} \theta^{2}+\frac{\mu_{i} \theta-\lambda \theta}{\delta_{i}+\theta} .
$$

For $q \geq 0$, there exists a $q$-scale function $W_{i}^{(q)}(x)$ for $x \in[0, \infty)$, which is defined to be the unique solution of

$$
\int_{0}^{+\infty} \mathrm{e}^{-\theta x} W_{i}^{(q)}(x) \mathrm{d} x=\frac{1}{\psi_{i}(\theta)-q}, \quad \theta>\Phi_{i}(q),
$$

where

$$
\Phi_{i}(q)=\sup \left\{s \geq 0: \psi_{i}(s)=q\right\}, \quad q \geq 0 .
$$

The $q$-scale functions in the special case of $q=0$ are called scale functions.

On the other hand, according to Corollary 4.2 of Egami and Yamazaki [10], a $q$-scale function $W_{i}^{(q)}(x)$ corresponding to a jump-diffusion process with negative exponential jumps has the following explicit expression

$$
W_{i}^{(q)}(x)=\frac{2}{\sigma_{i}^{2}} \sum_{k \in\{1,2\}} \frac{(-1)^{k}\left(\delta_{i}+\theta_{i k}^{(q)}\right)}{\left(\theta_{i 2}^{(q)}-\theta_{i 1}^{(q)}\right)\left(\theta_{i 3}^{(q)}-\theta_{i k}^{(q)}\right)}\left(\mathrm{e}^{\theta_{i 3}^{(q)}} x-\mathrm{e}^{\theta_{i k}^{(q)} x}\right), \quad q \geq 0,
$$

where $\theta_{i 1}^{(q)}, \theta_{i 2}^{(q)}$, and $\theta_{i 3}^{(q)}$ are three different roots of

$$
\psi_{i}(\theta)=q
$$

which is equivalent to

$$
\frac{1}{2} \theta^{3}+\left(\frac{\mu_{i}}{\sigma_{i}^{2}}+\frac{\delta_{i}}{2}\right) \theta^{2}+\left(\frac{\mu_{i}}{\sigma_{i}^{2}} \delta_{i}-\frac{q+\lambda}{\sigma_{i}^{2}}\right) \theta-\frac{q}{\sigma_{i}^{2}} \delta_{i}=0
$$

such that

$$
\theta_{i 1}^{(q)}<-\delta_{i}<\theta_{i 2}^{(q)}<0<\theta_{i 3}^{(q)}
$$

Obviously, the $q$-scale function $W_{i}^{(q)}(x)$ is twice continuously differentiable and an increasing function with $W_{i}^{(q)}(0)=0$. For more smoothness properties of the $q$-scale function; see Chan et al. [7]. It is also clear from (2.5)-(2.8) that $\Phi_{i}(q)=\theta_{i 3}^{(q)}>0$ and that $W_{i}^{(q)}(x)$ satisfies the following ordinary differential equation (ODE):

$$
\frac{1}{2} \sigma_{i}^{2} W^{(q)_{i} \prime \prime}(x)+\mu_{i} W_{i}^{(q) \prime \prime}(x)-(q+\lambda) W_{i}^{(q)}(x)+\lambda \delta_{i} \int_{0}^{x} W_{i}^{(q)}(x-y) \mathrm{e}^{-\delta_{i} y} \mathrm{~d} y=0 .
$$

\subsection{Bounds of the value function}

Assume for the moment that there is only a single regime, $E=\{i\}$. Then we are back in the setting of the jump-diffusion process with negative exponential jump that was investigated by Belhaj [5]. He showed that, if $\mu_{i}>0$, the optimal dividend strategy is a constant barrier strategy at the level $a_{i}^{*}$ such that

$$
g_{i}^{\left(r_{i}\right) \prime \prime}\left(a_{i}^{*}\right)=0
$$


where

$$
g_{i}^{\left(r_{i}\right)}(x)=\left(\gamma_{i 2}-\gamma_{i 3}\right) \exp \left(\theta_{i 1}^{\left(r_{i}\right)} x\right)+\left(\gamma_{i 3}-\gamma_{i 1}\right) \exp \left(\theta_{i 2}^{\left(r_{i}\right)} x\right)+\left(\gamma_{i 1}-\gamma_{i 2}\right) \exp \left(\theta_{i 3}^{\left(r_{i}\right)} x\right),
$$

with $\gamma_{i k}=\frac{1}{2} \sigma_{i}^{2}\left(\theta_{i k}^{\left(r_{i}\right)}\right)^{2}+\mu_{i} \theta_{i k}^{\left(r_{i}\right)}$ for $k=1,2,3$ and $\theta_{i 1}^{\left(r_{i}\right)}, \theta_{i 2}^{\left(r_{i}\right)}$, and $\theta_{i 3}^{\left(r_{i}\right)}$, are three roots of $\psi_{i}(\theta)=r_{i}$, that is, (2.8) with $q$ replaced by $r_{i}$ such that $\theta_{i 1}^{\left(r_{i}\right)}<-\delta_{i}<\theta_{i 2}^{\left(r_{i}\right)}<0<\theta_{i 3}^{\left(r_{i}\right)}$.

Indeed, it is worth mentioning that (2.8) and (2.11) imply that the optimal dividend barrier $a_{i}^{*}$ is finite and positive because

$$
\left.g_{i}^{\left(r_{i}\right) \prime \prime}(x)\right|_{x=0+}=\mu_{i}\left(\theta_{i 1}^{\left(r_{i}\right)}-\theta_{i 2}^{\left(r_{i}\right)}\right)\left(\theta_{i 2}^{\left(r_{i}\right)}-\theta_{i 3}^{\left(r_{i}\right)}\right)\left(\theta_{i 1}^{\left(r_{i}\right)}-\theta_{i 3}^{\left(r_{i}\right)}\right)<0,
$$

and

$$
\begin{aligned}
\left.g_{i}^{\left(r_{i}\right) \prime \prime}(x)\right|_{x=+\infty} & =\lim _{x \rightarrow+\infty}\left(\gamma_{i 1}-\gamma_{i 2}\right)\left(\theta_{i 3}^{\left(r_{i}\right)}\right)^{2} \exp \left(\theta_{i 3}^{\left(r_{i}\right)} x\right) \\
& =\lim _{x \rightarrow+\infty}\left(\theta_{i 3}^{\left(r_{i}\right)}\right)^{2}\left(\theta_{i 1}^{\left(r_{i}\right)}-\theta_{i 2}^{\left(r_{i}\right)}\right)\left[\frac{1}{2} \sigma_{i}^{2}\left(\theta_{i 1}^{\left(r_{i}\right)}+\theta_{i 2}^{\left(r_{i}\right)}\right)+\mu_{i}\right] \exp \left(\theta_{i 3}^{\left(r_{i}\right)} x\right) \\
& =\lim _{x \rightarrow+\infty} \frac{1}{2} \sigma_{i}^{2}\left(\theta_{i 3}^{\left(r_{i}\right)}\right)^{2}\left(\theta_{i 1}^{\left(r_{i}\right)}-\theta_{i 2}^{\left(r_{i}\right)}\right)\left(-\delta_{i}-\theta_{i 3}^{\left(r_{i}\right)}\right) \exp \left(\theta_{i 3}^{\left(r_{i}\right)} x\right) \\
& =+\infty \\
& >0 .
\end{aligned}
$$

On the other hand, due to the fact that $0<a_{i}^{*}<\infty$, it follows from Proposition 1 of Avram et al. [2] for the case of a spectrally negative Lévy process that the value function $V_{i}^{*}(x)$ can also be expressed below in terms of the $q$-scale function $W_{i}^{(q)}(x)$ for the case of the jump-diffusion process with negative exponential jumps, such that

$$
V_{i}^{*}(x)= \begin{cases}\frac{W_{i}^{\left(r_{i}\right)}(x)}{W_{i}^{\left(r_{i}\right) \prime}\left(a_{i}^{*}\right)}, & 0 \leq x \leq a_{i}^{*}, \\ x-a_{i}^{*}+\frac{W_{i}^{\left(r_{i}\right)}\left(a_{i}^{*}\right)}{W_{i}^{\left(r_{i}\right) \prime}\left(a_{i}^{*}\right)}, & x>a_{i}^{*} .\end{cases}
$$

From (2.11) and (2.12) we see that the value function and optimal dividend level are functions of not only the drift, jump intensity, and rate of discounting per unit of squared volatility but also average jump size. This observation leads us to expect that $V(x, i)$ is bounded above and below by the values $V_{+}(x)$ and $V_{-}(x)$ of firms operating in a more or less favourable environment, with unit volatility and drift, rate of discounting, jump intensity and average jump size

$$
\left(\frac{\mu_{+}}{\sigma_{+}^{2}}, \frac{r_{+}}{\sigma_{+}^{2}}, \frac{\lambda_{+}}{\sigma_{+}^{2}}, \delta_{+}\right)=\left(\max _{i \in E}\left(\frac{\mu_{i}}{\sigma_{i}^{2}}\right), \min _{i \in E}\left(\frac{r_{i}}{\sigma_{i}^{2}}\right), \min _{i \in E}\left(\frac{\lambda}{\sigma_{i}^{2}}\right), \max _{i \in E} \delta_{i}\right),
$$

and

$$
\left(\frac{\mu_{-}}{\sigma_{-}^{2}}, \frac{r_{-}}{\sigma_{-}^{2}}, \frac{\lambda_{-}}{\sigma_{-}^{2}}, \delta_{-}\right)=\left(\min _{i \in E}\left(\frac{\mu_{i}}{\sigma_{i}^{2}}\right), \max _{i \in E}\left(\frac{r_{i}}{\sigma_{i}^{2}}\right), \max _{i \in E}\left(\frac{\lambda}{\sigma_{i}^{2}}\right), \min _{i \in E} \delta_{i}\right),
$$

respectively.

It is very easy to see from the proofs of Proposition 2.1 and Lemma A.2 of Jiang and Pistorius [15] that the following two theorems regarding lower and upper bounds and Lipschitz continuity of the value function $V(x, i)$ also hold in our setting. 
Theorem 2.1. If $\mu_{-}>0$ then it follows that, for all $x \geq 0$ and $i \in E$,

$$
V_{-}(x) \leq V(x, i) \leq V_{+}(x) .
$$

Theorem 2.2. For $i \in E$ and $x \geq y \geq 0$, it holds that

$$
x-y \leq V(x, i)-V(y, i) \leq\left(1-\frac{W_{i}^{\left(\theta_{i}\right)}(y)}{W_{i}^{\left(\theta_{i}\right)}(x)}\right) V(x, i),
$$

where $\theta_{i}=r_{i}-q_{i i}$ and $W_{i}^{\left(\theta_{i}\right)}(\cdot)$ is given by (2.6) with $q=\theta_{i}$. Particularly, $V(x, i)$ is Lipschitz continuous in $x$.

The bounds in (2.13) will be applied in order to construct the optimal value function in Section 4.

\section{Dynamic programming equation and optimal dividend strategy}

\subsection{Dynamic programming equation}

Following the same arguments as in the proof of Proposition 2.2 of Jiang and Pistorius [15], we have the following dynamic programming equation for the value function $V(x, i)$ :

$$
V(x, i)=\sup _{D \in \mathcal{D}} \mathbb{E}_{x, i}\left[\int_{0}^{\tau \wedge \zeta} \mathrm{e}^{-\Lambda_{t}} \mathrm{~d} D_{t}+\mathrm{e}^{-\Lambda_{\tau \wedge \zeta}} V\left(U_{\tau \wedge \zeta}, Z_{\tau \wedge \zeta}\right)\right],
$$

where $\zeta$ denotes the first regime-switching epoch and $\Lambda_{t}=\int_{0}^{t} r\left(Z_{s}\right) \mathrm{d} s$.

This dynamic programming equation is associated with the following Hamilton-JacobiBellman (HJB) equation for the value function:

$$
\max \left\{g w(x, i)-r(i) w(x, i), 1-w^{\prime}(x, i)\right\}=0, \quad x>0, i \in E,
$$

where " " denotes the partial derivative with respect to $x$ and $g$ denotes the infinitesimal generator of $(X, Z)$ which acts on functions $w:[0, \infty) \times E \rightarrow[0, \infty)$ with $w(\cdot, i) \in C^{2}([0, \infty))$ for $i \in E$ as $g w(x, i)$, where

$$
\begin{aligned}
g w(x, i)= & \frac{1}{2} \sigma_{i}^{2} w^{\prime \prime}(x, i)+\mu_{i} w^{\prime}(x, i)+\sum_{j \neq i} q_{i j}[w(x, j)-w(x, i)] \\
& +\lambda \delta_{i} \int_{0}^{\infty}(w(x-y, i)-w(x, i)) \mathrm{e}^{-\delta_{i} y} \mathrm{~d} y,
\end{aligned}
$$

which is equivalent to

$$
\begin{aligned}
g w(x, i)= & \frac{1}{2} \sigma_{i}^{2} w^{\prime \prime}(x, i)+\mu_{i} w^{\prime}(x, i)-\left(\lambda-q_{i i}\right) w(x, i)+\sum_{j \neq i} q_{i j} w(x, j) \\
& +\lambda \delta_{i} \int_{0}^{x} w(x-y, i) \mathrm{e}^{-\delta_{i} y} \mathrm{~d} y,
\end{aligned}
$$

since our assumption of limited liability leads to $V(x-y, i)=0$ for any $y>x$, which indeed plays a crucial role in solving our optimal dividend control problem for the value function.

As a matter of fact, the following theorem shows that any sufficiently smooth supersolution of the HJB equation (3.2) dominates the value function. 
Theorem 3.1. Suppose that there exists a function $w=(w(\cdot, i), i \in E)$ such that $w(\cdot, i)$ for $i \in E$ are $C^{1}$ and piecewise $C^{2}$ functions on $(0, \infty)$ and satisfy the following condition, for $x>0$,

$$
\left\{\begin{array}{l}
g w(x, i)-r(i) w(x, i) \leq 0 \quad \text { in the distributional sense, } \\
w(0, i)=0, \quad w^{\prime}(x, i) \geq 1
\end{array}\right.
$$

Then we deduce that (i) $w(x, i) \geq V(x, i)$ for all $x \geq 0$ and $i \in E$ and that (ii) if, moreover, $w=V_{D}$ for some $D \in \mathscr{D}$, then $D$ is an optimal strategy such that $V \equiv w$.

Proof. (i) Fix an arbitrary $D \in \mathscr{D}$ and let $U_{t}$ be the corresponding risk process at time $t$. Applying a generalised form of Itô's lemma to the process $\left\{\mathrm{e}^{-\Lambda_{T \wedge \tau} w}\left(U_{T \wedge \tau}, Z_{T \wedge \tau}\right), T \geq 0\right\}$ yields

$$
\begin{aligned}
\mathrm{e}^{-\Lambda_{T \wedge \tau}} & w\left(U_{T \wedge \tau}, Z_{T \wedge \tau}\right)-w\left(U_{0}, Z_{0}\right)+\int_{0}^{T \wedge \tau} \mathrm{e}^{-\Lambda_{s}} \mathrm{~d} D_{s} \\
= & \int_{0}^{T \wedge \tau} \mathrm{e}^{-\Lambda_{s}}(g w-r w)\left(U_{s-}, Z_{s}\right) \mathrm{d} s+\int_{0}^{T \wedge \tau} \mathrm{e}^{-\Lambda_{s}}\left[1-w^{\prime}\left(U_{s-}, Z_{s-}\right)\right] \mathrm{d} D_{s}^{c} \\
& +\sum_{0 \leq s \leq T \wedge \tau} \mathrm{e}^{-\Lambda_{s}}\left[w\left(U_{s-}-\Delta D_{s}, Z_{s}\right)-w\left(U_{s-}, Z_{s-}\right)+\Delta D_{s}\right] \mathbf{1}_{\left\{\Delta D_{s}>0\right\}} \\
& +M_{T \wedge \tau},
\end{aligned}
$$

where $D_{t}=D_{t}^{c}+\sum_{0 \leq s \leq t} \Delta D_{s}, \mathbf{1}_{\left\{\Delta D_{s}>0\right\}}$ is an indicator function with value 1 for $\Delta D_{s}>0$ and with value 0 otherwise, and $M_{T \wedge \tau}$ is the local martingale with

$$
M_{t}=\int_{0}^{t} \mathrm{e}^{-\Lambda_{s}} \sigma\left(Z_{s-}\right) w^{\prime}\left(U_{s-}, Z_{s-}\right) \mathrm{d} W_{s}+\int \mathrm{e}^{-\Lambda_{s}}\left[w\left(U_{s-}, j\right)-w\left(U_{s-}, Z_{s-}\right)\right] \tilde{\pi}(\mathrm{d} s, \mathrm{~d} j) .
$$

Here the second integration is over $[0, t] \times[0, N]$ and $\tilde{\pi}=\pi-v$ is a compensated random measure (see, e.g. Jacod and Shiryaev [13, Section II.1.16] for more details of random measures), with $\pi(\mathrm{d} t, \mathrm{~d} j)=\sum_{s \geq 0} \mathbf{1}_{\left\{\Delta Z_{s}(\omega) \neq 0\right\}} \delta_{\left(s, Z_{s}(\omega)\right)}(\mathrm{d} t, \mathrm{~d} j)$, where $\delta_{(s, z)}$ denotes the Dirac measure at point $(s, z)$, and the compensator $v$ given by

$$
v(\mathrm{~d} t, \mathrm{~d} j)=p_{Z_{t-}}(j)\left[-q_{Z_{t-}, Z_{t-}}\right] \delta(\mathrm{d} j) \mathrm{d} t=q_{Z_{t-}, j} \delta(\mathrm{d} j) \mathrm{d} t, \quad j \in E,
$$

where $p_{Z_{t-}}(j)=\left(q Z_{t-}, j\right) /\left(-q_{Z_{t-}, Z_{t-}}\right)=\mathbb{P}\left(Z_{t}=j \mid Z_{t-}, \Delta Z_{t} \neq 0\right)$. Note that, from (3.4), since $M$ is bounded below and $M_{0}=0, M$ is a supermartingale with $E\left[M_{T \wedge \tau}\right] \leq 0$. In view of (3.2), $E\left[M_{T \wedge \tau}\right] \leq 0$, and the nonpositivity of the right-hand side of (3.4) excluding term $M_{T \wedge \tau}$, taking expectations yields

$$
w(x, i) \geq \mathbb{E}_{x, i}\left[\mathrm{e}^{-\Lambda_{T \wedge \tau}} w\left(U_{T \wedge \tau}, Z_{T \wedge \tau}\right)\right]+\mathbb{E}_{x, i}\left[\int_{0}^{T \wedge \tau} \mathrm{e}^{-\Lambda_{s}} \mathrm{~d} D_{s}\right] .
$$

By letting $T \rightarrow \infty$ and using the monotone convergence theorem and the fact that $w$ is nonnegative, we obtain $w(x, i) \geq V_{D}(x, i)$ and, hence, $w(x, i) \geq V(x, i)$.

(ii) The equality follows from the fact that $V_{D} \leq V$ by the definition of $V$ and that $V_{D} \geq V$ according to part (i). 


\subsection{Optimal dividend strategy}

The next step is to construct a candidate optimal solution by following the classical approach to solving optimal control problems. Due to the fact that $(U, Z)$ is a Markov process, we consider a modulated barrier strategy $D^{b} \in \mathscr{D}$ at level $b=(b(i), i \in E)$, as Definition 3.1 of Jiang and Pistorius [15], that pays out the overflow of the cash reserves above a regime-dependent level $b=(b(i), i \in E)$ such that

$$
\int_{0}^{\infty} \mathbf{1}_{\left\{U_{t}^{b}<b\left(Z_{t}\right)\right\}} \mathrm{d} D_{t}^{b}=0 \quad \text { and } \quad U_{t}^{b} \leq b\left(Z_{t}\right) \quad \text { for any } t \geq 0,
$$

where $U^{b}$ is the risk process (2.3) corresponding to $D^{b}$.

According to this strategy, dividends are only paid out when $U^{b}$ is at the barrier $b$, which indicates that the stochastic process $D^{b}$ is a local time at $b$. It is easy to verify that $D^{b}$ can be expressed in terms of a running supremum in the following explicit form

$$
D_{t}^{b}=0 \vee \sup _{0 \leq s \leq t}\left\{x+\int_{0}^{s} \mu\left(Z_{u}\right) \mathrm{d} u+\int_{0}^{s} \sigma\left(Z_{u}\right) \mathrm{d} W_{u}-\sum_{k=1}^{N_{s}} Y_{k}\left(Z_{s_{k}}\right)-b\left(Z_{s}\right)\right\},
$$

where $0 \leq s_{1}<s_{2}<\cdots<s_{N_{s}} \leq s \leq t$. Using the heuristic 'principle of $C^{2}$ fit' of singular control enables us to define candidate optimal levels as the solution to the system of equations

$$
V_{i}^{b \prime \prime}\left(b_{i}\right)=0, \quad i \in E,
$$

where $V_{i}^{b}(x)$ denotes the value function $V(x, i)$ with barrier strategy $b=\left(b_{i}, i \in E\right)$, if such a solution exists. As a matter of fact, (3.5) follows from Lemma 4.1 and Theorem 4.1 as will be seen later. If the drift is positive for all regimes, this candidate solution is indeed optimal as is shown later.

Theorem 3.2. Assume that $\mu_{i}>0$ for all $i \in E$. Then there exist levels $b^{*}=\left(b_{i}^{*}, i \in E\right)$ that solve the system (3.5), with $0<b_{i}^{*}<\infty$, and the following two assertions hold.

(i) The optimal value function $V$ is a classical solution of the HJB equation (3.2). Particularly, $V$ equals the unique solution $w=\{w(x, i), i \in E\}$ with $w(\cdot, i) \in C^{2}([0, \infty))$ of the following system

$$
\begin{cases}g w(x, i)-r(i) w(x, i)=0, & 0<x<b_{i}^{*}, \\ w_{i}(x)=x-b_{i}^{*}+w_{i}\left(b_{i}^{*}\right), & x \geq b_{i}^{*}, \\ w_{i}(0)=0, w_{i}^{\prime}\left(b_{i}^{*}\right)=1, & \end{cases}
$$

for $i \in E$, where $w_{i}(x)=w(x, i)$ and $g w(x, i)$ is given by (3.3).

(ii) The modulated barrier strategy at $b^{*}$ is indeed an optimal policy in (2.4).

In the following section we will present an iterative construction of the optimal value function in order to prove Theorem 3.2.

\section{An algorithm to calculate the value function}

To begin with, note that the value function $V^{b}$ corresponding to a modulated barrier strategy at dividend barrier $b=\left(b_{i}, i \in E\right)$ solves the following fixed point equation $V^{b}=T_{b}\left(V^{b}\right)$ in terms of the $q$-scale function $W_{i}^{(q)}$ given by (2.6). 
Theorem 4.1. For $i \in E$, it is true that $V^{b}=T_{b}\left(V^{b}\right)$, where

$$
T_{b}(f)(x, i)= \begin{cases}W_{i}^{\left(\theta_{i}\right)}(x) A_{i}^{f}\left(b_{i}\right)-\sum_{j \neq i} q_{i j} \int_{0}^{x} W_{i}^{\left(\theta_{i}\right)}(x-z) f(z, j) \mathrm{d} z, & 0 \leq x \leq b_{i}, \\ x-b_{i}+T_{b}(f)\left(b_{i}, i\right), & x \geq b_{i},\end{cases}
$$

for any $f:[0, \infty) \times E \rightarrow[0, \infty)$, where $\theta_{i}=r_{i}-q_{i i}$ and $A_{i}^{f}(\cdot)$ is given by

$$
A_{i}^{f}(y)=\frac{1}{W_{i}^{\left(\theta_{i}\right)^{\prime}}(y)}+\sum_{j \neq i} \frac{q_{i j}}{W_{i}^{\left(\theta_{i}\right)^{\prime}(y)}} \int_{0}^{y} W_{i}^{\left(\theta_{i}\right)^{\prime}}(y-z) f(z, j) \mathrm{d} z .
$$

The above result can be used to compute the value function $V^{b}$ of the modulated barrier strategy at $b$ by iterating the mapping $T_{b}: v \mapsto T_{b} v$. Let $\mathscr{B}$ denote the set

$$
\mathscr{B}=\left\{f: f_{i}(x):=f(x, i) \in C([0, \infty)), f(0, i)=0, \frac{f(x, i)}{1+|x|} \text { is bounded }\right\},
$$

and let $\|f\|=\max _{i \in E} \sup _{x \geq 0}|f(x, i)| /(1+|x|)$ for $f \in \mathscr{B}$.

Corollary 4.1. The mapping $T_{b}$ is a contraction on $\mathscr{B}$ with respect to the norm $\|\cdot\|$ and

$$
V^{b}(x, i)=\lim _{n \rightarrow \infty} T_{b}^{n}(f)(x, i)
$$

for $f \in \mathcal{B}$, where the convergence is in the sense of norm $\|\cdot\|$ and $T_{b}^{n}(f)=T_{b}\left(T_{b}^{n-1}(f)\right)$ for $n>1$ with $T_{b}^{1}=T_{b}$.

Proof of Theorem 4.1. Denote by $U_{t}^{i}=X_{t}^{i}-D_{t}^{i}$ the risk process with dividends $D_{t}^{i}$ paid according to a constant barrier strategy at $b_{i}$, with $X_{t}^{i}=\mu_{i} t+\sigma_{i} W_{t}-\sum_{k=1}^{N_{t}} Y_{k}(i)$. Let $\tau^{b}=$ $\inf \left\{t \geq 0: U_{t}^{b} \leq 0\right\}$ and $\tau^{i}=\inf \left\{t \geq 0: U_{t}^{i} \leq 0\right\}$ be the ruin times of $U_{t}^{b}$ and $U_{t}^{i}$, respectively. Denote by $\eta(a)$ an independent exponential random time with parameter $a$ and by $\zeta$ the first regime-switching epoch. Then $\left(U_{t}^{i}, t<\tau^{i} \wedge \eta\left(-q_{i i}\right)\right)$ is equal to $\left(U_{t}, Z_{0}=i, t<\tau^{b} \wedge \zeta\right)$ in distribution. Thus, the expectation $z_{1}(x, i)$ of the cumulative discounted dividends received before $\zeta$ is given by

$$
\begin{aligned}
z_{1}(x, i) & =\mathbb{E}_{x, i}\left[\int_{0}^{\tau^{b} \wedge \zeta} \mathrm{e}-\Lambda_{s} \mathrm{~d} D_{s}^{b}\right] \\
& =\mathbb{E}_{x}\left[\int_{0}^{\tau^{i}} \mathrm{e}^{-r_{i} s} \mathbf{1}_{\left\{s<\eta\left(-q_{i i}\right)\right\}} \mathrm{d} D_{s}^{i}\right] \\
& =\mathbb{E}_{x}\left[\int_{0}^{\tau^{i}} \mathrm{e}^{-\left(r_{i}-q_{i i}\right) s} \mathrm{~d} D_{s}^{i}\right] \\
& =\frac{W_{i}^{\left(\theta_{i}\right)}(x)}{W_{i}^{\left(\theta_{i}\right) \prime}\left(b_{i}\right)},
\end{aligned}
$$

where $\theta_{i}=r_{i}-q_{i i}$ and in the last line we use (2.12). 
On the other hand, the expectation $z_{2}(x, i)$ of the cumulative discounted dividends received after $\zeta$ has the following expression by the Markov property:

$$
\begin{aligned}
z_{2}(x, i) & =\mathbb{E}_{x, i}\left[\mathrm{e}^{-\Lambda_{\zeta \wedge \tau^{i}}} V^{b}\left(U_{\zeta \wedge \tau^{i}}^{b}, Z_{\zeta \wedge \tau^{i}}\right)\right] \\
& =\frac{-q_{i i}}{\theta_{i}} \mathbb{E}_{x}\left[V^{b}\left(U_{\eta\left(\theta_{i}\right)}^{i}, Z_{\eta\left(\theta_{i}\right)}\right) \mathbf{1}_{\left\{\eta\left(\theta_{i}\right)<\tau^{i}\right\}}\right] \\
& =\sum_{j \neq i} \frac{q_{i j}}{\theta_{i}} \int_{0}^{b_{i}} V_{j}^{b}(y) \mathbb{P}_{x}\left(U_{\eta\left(\theta_{i}\right)}^{i} \in \mathrm{d} y, \eta\left(\theta_{i}\right)<\tau^{i}\right) .
\end{aligned}
$$

Meanwhile, we have

$$
\begin{aligned}
& \mathbb{P}_{x}\left(U_{\eta\left(\theta_{i}\right)}^{i} \in \mathrm{d} y, \eta\left(\theta_{i}\right)<\tau^{i}\right) \\
& \quad=\mathbb{P}_{x-b_{i}}\left(b_{i}-\hat{Y}_{\eta\left(\theta_{i}\right)}^{i} \in \mathrm{d} y, \eta\left(\theta_{i}\right)<\tau_{b_{i}}\right) \\
& \quad=\theta_{i}\left[\frac{W_{i}^{\left(\theta_{i}\right)}(x) W_{i}^{\left(\theta_{i}\right) \prime}\left(b_{i}-y\right)}{W_{i}^{\left(\theta_{i}\right) \prime}\left(b_{i}\right)}-\mathbf{1}_{\{x \geq y\}} W_{i}^{\left(\theta_{i}\right)}(x-y)\right] \mathrm{d} y,
\end{aligned}
$$

where $\hat{Y}_{\eta\left(\theta_{i}\right)}^{i}=\sup _{s \leq \eta\left(\theta_{i}\right)}\left(X_{s}^{i}-b_{i}\right) \vee 0-\left(X_{\eta\left(\theta_{i}\right)}^{i}-b_{i}\right)$ started from $b_{i}-x$ under $\mathbb{P}_{x-b_{i}}$ is the reflected process of $X_{\eta\left(\theta_{i}\right)}^{i}-b_{i}$ at the supremum, $\tau_{b_{i}}=\inf \left\{t \geq 0: \hat{Y}_{t}^{i} \geq b_{i}\right\}$, the first equality is obtained by the fact that $\tau_{b_{i}}=\tau^{i}$ and $D_{\eta\left(\theta_{i}\right)}^{i}=\sup _{s \leq \eta\left(\theta_{i}\right)}\left(X_{s}^{i}-b_{i}\right) \vee 0$, and the second equality is derived from Theorem 1 of Pistorius [19]. Due to the fact that $V^{b}(x, i)=z_{1}(x, i)+z_{2}(x, i)$, we find the desired result.

The proof of Corollary 4.1 is omitted since it is the same as that of Corollary 4.1 of Jiang and Pistorius [15] except that the $\theta_{i}$-scale function in the case of Brownian motion with drift is replaced with the $\theta_{i}$-scale function given by (2.6) in the case of the jump-diffusion process with exponential jumps for $\theta_{i}=r_{i}-q_{i i}$.

\subsection{Iteration}

Our next step is to consider the following auxiliary control problem with payoff function $v$, which is to be received at the the first regime-switching epoch $\zeta$ :

$$
(U v)(x, i)=\sup _{D \in D} \mathbb{E}_{x, i}\left[\int_{0}^{\tau \wedge \zeta} \mathrm{e}^{-\Lambda_{t}} \mathrm{~d} D_{t}+\mathbf{1}_{\{\zeta<\tau\}} \mathrm{e}^{-\Lambda_{\zeta}} v\left(U_{\zeta}, Z_{\zeta}\right)\right]
$$

We can explicitly solve this singular control problem if $v$ is in the following set $\mathcal{C}$ of smooth and concave payoff functions,

$$
\mathcal{C}=\left\{v \in \mathscr{B}: v_{i} \text { is concave and increasing, } i \in E\right\} .
$$

Theorem 4.2. Suppose that $v \in \mathcal{C}$. Then $U v(\cdot, i) \in \mathcal{C}$ and $U v(\cdot, i) \in C^{2}[0, \infty)$ for $i \in E$ and the optimal strategy in (4.4) is a regime-switching barrier strategy at the dividend levels $b^{v}=\left(b_{i}^{v}, i \in E\right), 0<b_{i}^{v}<\infty$, given by

$$
b_{i}^{v}=\inf \left\{b \geq 0: A_{i}^{v}(x) \leq A_{i}^{v}(b) \text { for all } x \geq 0\right\},
$$

where $A^{v}$ is defined by (4.2) with $f=v$. Moreover, $U v(\cdot, i)=T_{b^{v}}(v)(\cdot, i) \in \mathcal{C}$. 
Since $V_{ \pm}(x)$ in the absence of regime switching are in the set $\mathcal{C} \subset \mathscr{B}$ and the mapping $U: v \mapsto U v$ preserves both concavity and smoothness, we can invoke Theorem 4.2 to find the optimal dividend barrier $b^{v}$ in the iterative algorithm as given by Jiang and Pistorius [15]. Begin with $n=0$ and $v=v_{0}$ by taking $v_{0}=V_{-} \in \mathcal{C}$ or $v_{0}=V_{+} \in \mathcal{C}$ in Theorem 2.1 and then iteratively use the following two steps until certain accuracy is reached:

(1) solve $b^{v}=\left(b_{i}^{v}, i \in E\right)$ in (4.5);

(2) set $v \leftarrow T_{b^{v}}(v), n \leftarrow n+1$, and $v_{n} \leftarrow v$, and then return to step (1).

Simultaneously, the above iteration also produces the value function with enough accuracy as long as the following theorem is true, that is, the sequence $\left(v_{n}\right)$ generated by this way approaches value function $V$ as $n \rightarrow \infty$.

Theorem 4.3. Let $v_{0}^{ \pm} \in \mathcal{C}$ and $v_{n}^{ \pm}=U v_{n-1}^{ \pm}$for $n \geq 1$. If $v_{0}^{-} \leq V \leq v_{0}^{+}$then we deduce that $v_{n}^{-} \leq V \leq v_{n}^{+}$and that

$$
V(x, i)=\lim _{n \rightarrow \infty} v_{n}^{+}(x, i)=\lim _{n \rightarrow \infty} v_{n}^{-}(x, i),
$$

where the convergence is with respect to norm $\|\cdot\|$. Particularly, $V$ is concave.

As a matter of fact, we will show in the following subsection that $U$ is a contraction on $\mathcal{C}$. It is now obvious that Theorem 3.2(i) is a direct consequence of the dynamic programming equation (3.1) and Theorem 4.2.

\subsection{Proofs}

Along the lines of Jiang and Pistorius [15], we split the proofs of Theorems 4.2 and 4.3 into several steps. The first step is to verify the following lemma about the existence of optimal barrier levels that the $b_{i}^{v}$ defined above are positive and finite, which is a matter of straightforward calculations using the closed-form expression (2.6) for $W_{i}^{\left(\theta_{i}\right)}(x)$.

Lemma 4.1. Suppose that $v \in \mathcal{C}$. Then $b \mapsto A_{i}^{v}(b)$ reaches its maximum at some $b_{i} \in(0, \infty)$, satisfying $T_{b}(v)_{i}^{\prime \prime}\left(b_{i}\right):=\left.\left(\partial^{2} / \partial x^{2}\right) T_{b}(v)(x, i)\right|_{x=b_{i}}=0, i \in E$. Particularly, $T_{b^{v}}(x, i)$ is $C^{2}$ in $x \in[0, \infty)$ for $i \in E$.

Proof. Due to the continuity of $A_{i}^{v}(b), A_{i}^{v}(b)$ reaches its maximum at some $b \in[0, \infty]$ and its derivative is given by

$$
W_{i}^{\left(\theta_{i}\right) \prime}(b) A_{i}^{v \prime}(b)=-\frac{W_{i}^{\left(\theta_{i}\right) \prime \prime}(b)}{W_{i}^{\left(\theta_{i}\right) \prime}(b)}+\sum_{j \neq i} \int_{0}^{b} q_{i j} v_{j}^{\prime}(y) k_{i}(b-y, b) \mathrm{d} y,
$$

where $k_{i}(y, b)=W_{i}^{\left(\theta_{i}\right) \prime}(y)-W_{i}^{\left(\theta_{i}\right)}(y) W_{i}^{\left(\theta_{i}\right) \prime \prime}(b) / W_{i}^{\left(\theta_{i}\right) \prime}(b)$. From (2.6) and (2.9), it is easy to show that $W_{i}^{\left(\theta_{i}\right) \prime \prime}(b) / W_{i}^{\left(\theta_{i}\right) \prime}(b)$ tends to $\theta_{i 3}^{(q)}>0$ with $q=\theta_{i}$ as $b \rightarrow \infty$, and, hence, the following estimate holds:

$$
\lim _{b \rightarrow \infty} k_{i}(y, b)=\frac{2}{\sigma_{i}^{2}} \sum_{k \in\{1,2\}} \frac{(-1)^{k}\left(\delta_{i}+\theta_{i k}^{(q)}\right)}{\left(\theta_{i 2}^{(q)}-\theta_{i 1}^{(q)}\right)\left(\theta_{i 3}^{(q)}-\theta_{i k}^{(q)}\right)}\left(\left(\theta_{i 3}^{(q)}-\theta_{i k}^{(q)}\right) \mathrm{e}^{\theta_{i k}^{(q)} y}\right) \leq 2 \sigma_{i}^{-2} \mathrm{e}^{\theta_{i 2}^{(q)} y}
$$

for $q=\theta_{i}$. From the dominated convergence theorem, it then follows that $W_{i}^{\left(\theta_{i}\right) \prime}(b) A_{i}^{v \prime}(b)$ approaches $-\theta_{i 3}^{(q)}<0$ with $q=\theta_{i}$ as $b \rightarrow \infty$. Because $A_{i}^{v \prime}(0+)=\mu_{i}>0$ as a result of $W_{i}^{\left(\theta_{i}\right) \prime}(0+)=2 / \sigma_{i}^{2}$ and $W_{i}^{\left(\theta_{i}\right) \prime \prime}(0+)=-4 \mu_{i} / \sigma_{i}^{4}, A_{i}^{v}(b)$ reaches its maximum on $(0, \infty)$. 
Therefore, $A_{i}^{v \prime}\left(b_{i}\right)=0$, implying that $\left(T_{b} v\right)_{i}^{\prime \prime}\left(b_{i}\right)=0$ by the definition of $T_{b} v$ and $\left(T_{b} v\right)_{i}^{\prime \prime}\left(b_{i}\right)=$ $W_{i}^{\left(\theta_{i}\right) \prime}(b) A_{i}^{v \prime}(b)$. Due to the fact that $T_{b} v^{\prime \prime}(x, i)=0$ for $x>b_{i}$, it follows that $T_{b^{v}}(\cdot, i) \in$ $C^{2}[0, \infty)$ for $i \in E$.

The next step is to verify the preservation of concavity in the following lemma that the value function, which corresponds to a barrier strategy at $b^{v}$ with a concave payoff function $v(\cdot, i)$, is also concave.

Lemma 4.2. If $v \in \mathcal{C}$, it is then true that $T_{b^{v}}(v) \in \mathcal{C}$.

Proof. We first assume that $v \in \mathcal{C} \cap C^{2}[0, \infty)$, and write $b$ instead of $b^{v}$ to simplify the notation. Because of the smoothness of $v$ and the definition of $w_{i}(x):=\left(T_{b^{v}} v\right)(x, i)$, it is derived from (2.6) and (4.1) that for, $x \in\left(0, b_{i}\right)$,

$$
\begin{aligned}
w_{i}^{\prime}(x) & =W_{i}^{\left(\theta_{i}\right) \prime}(x) A_{i}^{v}\left(b_{i}\right)-\sum_{j \neq i} q_{i j} \int_{0}^{x} W_{i}^{\left(\theta_{i}\right) \prime}(x-y) v(y, j) \mathrm{d} y, \\
w_{i}^{\prime \prime}(x)= & W_{i}^{\left(\theta_{i}\right) \prime \prime}(x) A_{i}^{v}\left(b_{i}\right) \\
& -\sum_{j \neq i} q_{i j}\left[W_{i}^{\left(\theta_{i}\right) \prime}(0+) v(x, j)+\int_{0}^{x} W_{i}^{\left(\theta_{i}\right) \prime \prime}(x-y) v(y, j) \mathrm{d} y\right] .
\end{aligned}
$$

From the above two expressions, (2.6), and $v \in C^{2}[0, \infty)$, we obtain that $\left.w_{i}\right|_{\left(0, b_{i}\right)} \in C^{4}\left(0, b_{i}\right)$. Moreover, we have $w_{i}^{\prime}\left(b_{i}\right)=1$ in view of the above expressions and (4.1), and have $w_{i}^{\prime \prime}\left(b_{i}\right)=0$ according to Lemma 4.1. Therefore, $w_{i}$ is $C^{2}[0, \infty)$.

Therefore, in view of $W_{i}^{\left(\theta_{i}\right) \prime}(0+)=2 / \sigma_{i}^{2}$ and the fact that $W_{i}^{\left(\theta_{i}\right) \prime}(x)$ satisfies (2.10) with $q=\theta_{i}$, multiplying (4.1), (4.6), and (4.7) by $-\left(\lambda+\theta_{i}\right), \mu_{i}$, and $\sigma_{i}^{2} / 2$, respectively, and adding them leads to

$$
\begin{aligned}
f_{i}^{v}(x):= & \frac{\sigma_{i}^{2}}{2} w_{i}^{\prime \prime}(x)+\mu_{i} w_{i}^{\prime}(x)-\left(\lambda+r_{i}-q_{i i}\right) w_{i}(x)+\lambda \delta_{i} \int_{0}^{x} w_{i}(x-y) \mathrm{e}^{-\delta_{i} y} \mathrm{~d} y \\
& +\sum_{j \neq i} q_{i j} v_{j}(x) \\
= & 0, \quad x \in\left(0, b_{i}\right),
\end{aligned}
$$

with boundary conditions $w_{i}(0)=0, w_{i}^{\prime}\left(b_{i}\right)=1$, and $w_{i}^{\prime \prime}\left(b_{i}\right)=0$. Because $w_{i}(x) \geq 0$ for $x>0$ and $w_{i}(0)=0$, it follows that $w_{i}^{\prime}(0+) \geq 0$. Moreover, the continuity of $w_{i}, w_{i}(0)=0$, and $v_{i}(0)=0$ indicate that

$$
\sigma_{i}^{2} w_{i}^{\prime \prime}(0+)+2 \mu_{i} w_{i}^{\prime}(0+)=0,
$$

so that $w_{i}^{\prime \prime}(0+)<0$ as a result of $\mu_{i}>0$.

Write $\xi_{i}(x)=w_{i}^{\prime \prime}(x)$ for $x>0$ and $\xi_{i}(0)=w_{i}^{\prime \prime}(0+)$. Since $w_{i}(x) \in C^{4}\left(0, b_{i}\right)$ as a result of the assumptions, differentiating twice the first equation of the original system (3.6), it follows that $\xi_{i}(x)$ satisfies the following ODE:

$$
\left\{\begin{array}{l}
\frac{\sigma_{i}^{2}}{2} \xi_{i}^{\prime \prime}(x)+\mu_{i} \xi_{i}^{\prime}(x)-\left(\lambda+r_{i}-q_{i i}\right) \xi_{i}(x) \\
\quad+\sum_{j \neq i} q_{i j} v_{j}^{\prime \prime}(x)+\lambda \delta_{i} \int_{0}^{x} \xi_{i}(x-y) \mathrm{e}^{-\delta_{i} y} \mathrm{~d} y=0, \quad x \in\left(0, b_{i}\right), \\
\xi_{i}(0)<0, \quad \xi_{i}\left(b_{i}\right)=0, \quad \xi_{i}(x)=0, \quad x>b_{i} .
\end{array}\right.
$$


Applying Itô's lemma to $\mathrm{e}^{-\theta_{i} t} \xi_{i}\left(X_{t}\right)$ from 0 to $T^{i}$ yields the stochastic representation for $\xi$,

$$
\xi_{i}(x)=\mathbb{E}_{x}\left[\mathrm{e}^{-\theta_{i} T^{i}} \xi_{i}\left(X_{T^{i}}\right)\right]+\sum_{j \neq i} q_{i j} \mathbb{E}_{x}\left[\int_{0}^{T^{i}} \mathrm{e}^{-\theta_{i} t} v_{j}^{\prime \prime}\left(X_{t}\right) \mathrm{d} t\right]
$$

where $\theta_{i}=\left(r_{i}-q_{i i}\right)$ and $T^{i}=\inf \left\{t \geq 0: X_{t}^{i} \notin\left(0, b_{i}\right)\right\}$. Thus, from the fact that $\xi_{i}\left(X_{T^{i}}\right) \leq 0$ and $v_{j}^{\prime \prime}(x) \leq 0$, it readily follows that $\xi_{i}(x)$ is nonpositive for all $x \in\left(0, b_{i}\right)$ and $i \in E$. As a result, it follows that $x \mapsto w_{i}(x)$ is increasing and concave on $[0, \infty)$.

Now assume that $v \in \mathcal{C}$ and denote by $v_{n} \in \mathcal{C} \cap C^{2}[0, \infty)$ a sequence that increases to $v$ pointwise. Then $T_{b^{v}}(v)(x, i)=\lim _{n \rightarrow \infty} T_{b^{v}}\left(v_{n}\right)(x, i)$, and the concavity of $T_{b^{v}}(v)$ is deduced from the fact that the pointwise limit of concave functions is concave.

We then verify the following lemma which concerns the optimality of barrier strategies that the modulated barrier strategy at $b^{v}$ is optimal for the problem (4.4).

Lemma 4.3. For $v \in \mathcal{C}$, it holds that $T_{b^{v}}(v)(x, i)=U v(x, i)$ for $x>0, i \in E$.

Proof. Let $w_{i}(x)=T_{b^{v}}(v)(x, i)$ and define $f_{i}^{v}(x)$ as in (4.8). In order to verify that $f_{i}^{v}(x) \leq 0$ for $x>b_{i}$, we first prove the following claim that

$$
f_{i}^{v \prime}\left(b_{i}+\right) \leq 0, \quad i \in E .
$$

From the fact that $w_{i}^{\prime \prime}\left(b_{i}\right)=0$ as a consequence of Lemma 4.1 and that $w_{i}^{\prime \prime}(x) \leq 0$ for $x<b_{i}$ as a result of the concavity of the $w_{i}$, it follows that $w_{i}^{\prime \prime \prime}\left(b_{i}-\right) \geq 0$. Because $w_{i}^{\prime}(x)$ and $w_{i}^{\prime \prime}(x)$ are both continuous at $x=b_{i}$ and $w_{i}^{\prime \prime \prime}\left(b_{i}+\right)=0$, it follows that $f_{i}^{v \prime}\left(b_{i}-\right) \geq f_{i}^{v \prime}\left(b_{i}+\right)$ as a consequence of the concavity of $v_{j}(x)$ for any $j \in E$. Finally, differentiating the two sides of $f_{i}^{v}(x) \equiv 0$ in (4.8) for $x \in\left(0, b_{i}\right)$ indicates that $f_{i}^{v \prime}\left(b_{i}-\right)=0$ and, hence, (4.9) follows.

Observe that

$$
f_{i}^{v \prime}(x)=-\left(\lambda+r_{i}-q_{i i}\right)+\lambda \delta_{i} \int_{0}^{x} w_{i}^{\prime}(x-y) \mathrm{e}^{-\delta_{i} y} \mathrm{~d} y+\sum_{j \neq i} q_{i j} v_{j}^{\prime}(x), \quad x>b_{i} .
$$

In view of (4.9), the concavity of both $v$ and $w_{i}$ yields $f_{i}^{v \prime}(x) \leq f_{i}^{v \prime}\left(b_{i}+\right) \leq 0$ for $x>b_{i}$, indicating that $f_{i}^{v}(x) \leq 0$ for $x \geq b_{i}$ by the fact that $f_{i}^{v}\left(b_{i}\right)=0$.

Since $w_{i}$ are concave and twice continuously differentiable, and satisfy (4.8), the assertion of the lemma follows from an argument similar to that used in the proof of Theorem 3.1. Fix an arbitrary $D \in \mathscr{D}$ and denote by $U_{t}$ the corresponding risk process at $t$. Applying a generalised form of Itô's lemma to the stochastic process $\left\{\mathrm{e}^{-\Lambda_{T \wedge \tau}} w\left(U_{T \wedge \tau}, Z_{T \wedge \tau}\right), T \geq 0\right\}$, taking expectations, and using the proved result that $f_{i}^{v}(x) \leq 0$ as in the proof of Theorem 3.1, we have

$$
w(x, i) \geq \mathbb{E}_{x, i}\left[\mathrm{e}^{-\Lambda_{T \wedge \tau}} w\left(U_{T \wedge \tau}, Z_{T \wedge \tau}\right)+\mathbf{1}_{\{\zeta<T \wedge \tau\}} \mathrm{e}^{-\Lambda_{\zeta}} v\left(U_{\zeta}, Z_{\zeta}\right)+\int_{0}^{T \wedge \tau \wedge \zeta} \mathrm{e}^{-\Lambda_{s}} \mathrm{~d} D_{s}\right] .
$$

By letting $T \rightarrow \infty$ and using the monotone convergence theorem and the fact that $v$ and $w$ are nonnegative and that $f_{i}^{v}(x) \leq 0$, we deduce that $w(x, i) \geq U v(x, i)$. Since the barrier strategy at level $b^{v}$ is in set $\mathscr{D}$, it holds that $U v(x, i) \geq w(x, i)$ and, hence, $w(x, i)=U v(x, i)$.

The convergence of the iteration procedure shown above is a direct result of the following contraction property of $U v$. 
Lemma 4.4. The mapping $v \mapsto U v$ is a contraction on $\mathcal{C}$ with respect to norm $\|\cdot\|$.

Proof. Since, according to Lemmas 4.2 and 4.3, $U v(x, i)=\sup _{b} T_{b}(v)(x, i)=\left(T_{b^{v}} v\right)(x, i)$ and $U v \in \mathcal{C}$ for $v \in \mathcal{C}$, we have, for $v, w \in \mathcal{C}$,

$$
\|U v-U w\| \leq \sup _{b}\left\|T_{b} v-T_{b} w\right\| \leq C\|v-w\|,
$$

where $C=\max _{i} \sum_{j \neq i}\left(q_{i j} / \theta_{i}\right) \in(0,1)$ and the second inequality follows from

$$
\left\|T_{b} v-T_{b} w\right\| \leq \max _{i \in E, x \in\left[0, b_{i}\right]} \sum_{j \neq i} \frac{q_{i j}}{\theta_{i}}\left(1-\mathbb{E}_{x, i}\left(\mathrm{e}^{-\theta_{i} \tau^{i}}\right)\right)\|v-w\| \leq C\|v-w\|,
$$

where we use (4.3) and the facts that $\eta\left(\theta_{i}\right)$ is an exponential random time with parameter $\theta_{i}$ and that $U_{\eta\left(\theta_{i}\right)}^{i} \in\left(0, b_{i}\right]$ for $\eta\left(\theta_{i}\right)<\tau^{i}$ to obtain

$$
\int_{0}^{b_{i}} \theta_{i}\left[\frac{W_{i}^{\left(\theta_{i}\right)}(x) W_{i}^{\left(\theta_{i}\right) \prime}\left(b_{i}-y\right)}{W_{i}^{\left(\theta_{i}\right) \prime}\left(b_{i}\right)}-\mathbf{1}_{\{x \geq y\}} W_{i}^{\left(\theta_{i}\right)}(x-y)\right] \mathrm{d} y=1-\mathbb{E}_{x, i}\left(\mathrm{e}^{-\theta_{i} \tau^{i}}\right) \leq 1
$$

for $\tau^{i}=\inf \left\{t \geq 0: U_{t}^{i} \leq 0\right\}$, as defined in the proof of Theorem 4.1. Thus, $U$ is a contraction on $\mathcal{C}$.

Theorem 4.2 directly follows from Lemma 4.1 and Lemma 4.3. From the dynamic programming equation and the definition of $U$, it readily follows that $U v \leq V \leq U w$ if $v \leq V \leq w$. In particular, taking $v=v_{0}^{-}$and $w=v_{0}^{+}$and then applying the former inequality repeatedly yields the inequality $v_{n}^{-} \leq V \leq v_{n}^{+}$. It follows from Lemma 4.4 that both $v_{n}^{+}$and $v_{n}^{-}$converge to the unique fixed point of $U$, which is, thus, equal to the value function $V$. Note that, in view of Lemma 4.2 and the fact that $v_{0}^{ \pm} \in \mathcal{C}, v_{n}^{ \pm}$are concave. As a result, $V$, a pointwise limit of concave functions, is also concave. This completes the proof of Theorem 4.3.

\section{Conclusions}

In this paper we have used the fixed point theorem and stochastic control theory to show that, in the setting of a Markov-modulated jump-diffusion process followed by cash reserves of a financial corporation with limited liability, the optimal dividend policy is a modulated barrier strategy at a dividend level as a function of the current regime. That is to say, the optimal dividend policy, which maximises the expectation of the cumulative present value of the dividends paid until the moment of bankruptcy, is to pay out as dividends the overflow of cash reserves above a certain optimal threshold, where this threshold may jump up or down exactly at the moment of regime shifts. In addition, we have also presented a contraction algorithm, an extension to that of Jiang and Pistorius [15], to calculate both the value function and optimal threshold levels in different regimes. However, we do not consider the case of negative drifts. The problem of optimal dividend policy in the case of negative drifts in some regimes and positive drifts in other regimes is challenging and worthy of further research. It is also noteworthy that the optimality of a barrier strategy in the dividend distribution problems is not clear in general and is in fact not true in the case of general jump-distributions, as shown, for example, by Avram et al. [3] and Azcue and Muler [4]. So, the inclusion of more general jump structures may be another further research topic. 


\section{Acknowledgements}

The author expresses his gratitude to the editor and the anonymous referee who provided valuable suggestions to improve the paper's organization and proofs significantly.

\section{References}

[1] Asmussen, S. and Taksar, M. (1997). Controlled diffusion models for optimal dividend pay-out. Insurance Math. Econom. 20, 1-15.

[2] Avram, F., Palmowski, Z. and Pistorius, M. R. (2007). On the optimal dividend problem for a spectrally negative Lévy process. Ann. Appl. Prob. 17, 156-180.

[3] Avram, F., Palmowski, Z. and Pistorius, M. R. (2014). On Gerber-Shiu functions and optimal dividend distribution for a Lévy risk-process in the presence of a penalty function. Preprint. Available at http://arxiv.org/abs/1110.4965.

[4] Azcue, P. ANd Muler, N. (2005). Optimal reinsurance and dividend distribution policies in the CramérLundberg model. Math. Finance 15, 261-308.

[5] BelhaJ, M. (2010). Optimal dividend payments when cash reserves follow a jump-diffusion process. Math. Finance 20, 313-325.

[6] Cadenillas, A., Sarkar, S. and Zapatero, F. (2007). Optimal dividend policy with mean-reverting cash reservoir. Math. Finance 17, 81-109.

[7] Chan, T., Kyprianou, A. E. And Savov, M. (2011). Smoothness of scale functions for spectrally negative Lévy processes. Prob. Theory Relat. Fields 150, 691-708.

[8] Décamps, J.-P. and Villeneuve, S. (2007). Optimal dividend policy and growth option. Finance Stoch. 11, 3-27.

[9] De Finetri, B. (1957). Su un'impostazione alternativa della teoria colletiva del rischio. Trans. XV Internat. Congr. Actuaries 2, 433-443.

[10] Egami, M. and Yamazaki, K. (2014). Phase-type fitting of scale functions for spectrally negative Lévy processes. J. Comput. Appl. Math. 264, 1-22.

[11] Hamilton, J. D. (1989). A new approach to the economic analysis of nonstationary time series and the business cycle. Econometrica 57, 357-384.

[12] Hamilton, J. D. (1990). Analysis of time series subject to changes in regime. J. Econometrics 45, 39-79.

[13] Jacod, J. And Shiryaev, A. N. (2003). Limit Theorems for Stochastic Processes, 2nd edn. Springer, Berlin.

[14] Jeanblanc-Picqué, M. and Shiryaev, A. N. (1995). Optimization of the flow of dividends. Russian Math. Surveys 50, 257-278.

[15] Jiang, Z. And Pistorius, M. (2012). Optimal dividend distribution under Markov regime switching. Finance Stoch. 16, 449-476.

[16] Kyprianou, A. E. and Palmowski, Z. (2007). Distributional study of de Finetti's dividend problem for a general Lévy insurance risk process. J. Appl. Prob. 44, 428-443.

[17] Loeffen, R. L. (2008). On optimality of the barrier strategy in de Finetti's dividend problem for spectrally negative Lévy processes. Ann. Appl. Prob. 18, 1669-1680.

[18] LøKKa, A. AND Zervos, M. (2008). Optimal dividend and issuance of equity policies in the presence of proportional costs. Insurance Math. Econom. 42, 954-961.

[19] Pistorius, M. R. (2004). On exit and ergodicity of the spectrally one-sided Lévy process reflected at its infimum. J. Theoret. Prob. 17, 183-220.

[20] Sotomayor, L. R. AND CADENILlas, A. (2011). Classical and singular stochastic control for the optimal dividend policy when there is regime switching. Insurance Math. Econom. 48, 344-354.

[21] WeI, J., WANG, R. AND YANG, H. (2012). On the optimal dividend strategy in a regime-switching diffusion model. Adv. Appl. Prob. 44, 886-906.

[22] Wei, J., YANG, H. AND WANG, R. (2010). Classical and impulse control for the optimization of dividend and proportional reinsurance policies with regime switching. J. Optimization Theory Appl. 147, 358-377.

[23] WeI, J., YANG, H. AND WANG, R. (2010). Optimal reinsurance and dividend strategies under the Markovmodulated insurance risk model. Stoch. Anal. Appl. 28, 1078-1105.

[24] ZHU, J. (2014). Singular optimal dividend control for the regime-switching Cramér-Lundberg model with credit and debit interest. J. Computational Appl. Math. 257, 212-239.

[25] Zhu, J. AND CHEN, F. (2013). Dividend optimization for regime-switching general diffusions. Insurance Math. Econom. 53, 439-456. 